
\title{
Research Square \\ Feasibility study on use of near infrared spectroscopy for rapid and non-destructive determination of gossypol content in intact cottonseeds
}

\section{Cheng Li}

Zhejiang University

\section{Bangsong Su}

Zhejiang University

Tianlun Zhao

Zhejiang University

Cong Li

Zhejiang University

Jinhong Chen

Zhejiang University

Shuijin Zhu ( $\nabla$ shjzhu@zju.edu.cn )

Zhejiang University https://orcid.org/0000-0001-6209-9630

\section{Research}

Keywords: intact cottonseed, chemometrics, gossypol, near infrared spectroscopy

Posted Date: January 15th, 2021

DOI: https://doi.org/10.21203/rs.3.rs-53311/v2

License: (c) This work is licensed under a Creative Commons Attribution 4.0 International License.

Read Full License 


\section{Abstract}

Background: Gossypol found in cottonseeds is toxic to human beings and monogastric animals and is a primary parameter for the integrated utilization of cottonseed products. It is usually determined by the techniques relied on complex pretreatment procedures and the samples after determination cannot be used in breeding program, so it is of great importance to predict the gossypol content in cottonseeds rapidly and non-destructively to substitute the traditional analytical method.

Results: Gossypol content in cottonseeds was investigated by near-infrared spectroscopy (NIRS) and High-performance liquid chromatography (HPLC). Partial least squares regression, combined with spectral pretreatment methods including Savitzky-Golay smoothing, standard normal variate, multiplicative scatter correction, and first derivate, were tested for optimizing the calibration models. NIRS technique was efficient in predicting gossypol content in intact cottonseeds, as revealed by the rootmean-square error of cross-validation (RMSECV), root-mean-square error of prediction (RMSEP), coefficient for determination of prediction $\left(R_{\mathrm{p}}{ }^{2}\right)$, and residual predictive deviation (RPD) values for all models, being $0.05-0.07,0.04-0.06,0.82-0.92$, and 2.3-3.4, respectively. The optimized model pretreated by Savitzky-Golay smoothing + standard normal variate + first derivate resulted in good determination of gossypol content in intact cottonseeds.

Conclusions: Near infrared spectroscopy coupled with different spectral pretreatments and PLS regression has exhibited the feasibility in predicting gossypol content in intact cottonseeds, rapidly and non-destructively. It could be used as an alternative method to substitute for traditional one to determine the gossypol content in intact cottonseeds.

\section{Introducton}

Cotton (Gossypium. spp) is one of the important industrial and economic crops (Sunilkumar et al. 2006). Cottonseed, the main by-product of cotton production, can be used to produce food, animal feed, and other products. Cottonseed contains many kinds of nutrients, including proteins, oils, fatty acids, and amino acids, making it a potential food resource for human beings with the rapid growth of global population (Sawan et al. 2006). However, the Gossypium species are characterized by the presence of gossypol, which is toxic to human beings and monogastric animals (Lordelo et al. 2005), such that the utilization of cottonseed products is limited.

Gossypol, 1, 1', 6, 6', 7, 7'-hexahydroxy-5, 5'-diisopropyl-3, 3'-dimethyl-(2, 2' binaphthalene)-8, 8'dicarbaldehyde, is a terpenoid compound that helps cotton defend against biotic stress (Kong et al. 2010; Lin et al. 1993; Blanco et al. 1983). Due to the toxicity of gossypol, breeding for both lower gossypol content in cottonseeds and higher gossypol content in cotton plants has been practiced in many cottonplanting countries. The cottonseed breeding work often requires analyzing a large number of cottonseed samples to measure gossypol content. Conventionally, gossypol content is assayed by UV spectrophotometry which not only involves reagents with great toxicity, but also is not accurate and 
reliable. Despite offering a high level of accuracy and sensitivity, HPLC is usually costly and timeconsuming. In addition, both classical analytical methods cause undesired destruction of the testing samples which frequently needed to be planted in cotton breeding program. So, a rapid and nondestructive method for gossypol determination is required.

Near infrared (NIR) spectroscopy combined with chemometrics is a rapid, convenient, and environmentally-friendly analytical technique in the quality analysis for crops (Sohn et al. 2008; Huang et al. 2013; Weinstock et al. 2006; Rosales et al. 2011; Bellato et al. 2011; Bala et al. 2013; Hacisalihoglu et al. 2010; Mendoza et al. 2018; Lee et al. 2017; Tierno et al. 2016; Yang et al. 2008; Lin et al. 2013; Kovalenko et al. 2006; Fassio et al. 2004). Although the NIR calibration model for determining gossypol content in cotton powder was developed (Li et al. 2017), it could not be use to non-destructively analyze gossypol content in intact cottonseeds, especially in breeding programs where the genetic materials from genetic modification or cross breeding have a limited availability. It is a challenge to determine gossypol content in intact cottonseeds by NIR, because (i) cottonseed being bigger than other crop seeds, so large voids are left between packed samples in sample cells; (ii) some of immature and wizened cottonseeds can be mixed in the samples, which can introduce irrelevant information into the spectra data; and (iii) the tough and thick shell of cottonseed can impact the penetration of NIR light and result in a lower S/N ratio and poor information. Because of these factors, the spectral data of intact cottonseeds are far more complex than that of other crop seeds, which may contain a large amount of useless and uncorrelated information such as noise and background. To overcome these difficulties, sophisticated chemometric methods are applied to extract useful information from NIR spectra and calibrate robust models for gossypol content in intact cottonseeds. Essentially, these include regression methods such as principal component regression (PCR) (Xie et al. 1997), partial least squares (PLS) (Haaland et al. 1988), support vector machines (SVM) (Nie et al. 2008), least squares support vector machines (LS-SVM) (Shao et al. 2012), and artificial neural networks (ANN) (Makinoa et al. 2010), coupled with spectral pretreatments such as standard normal variate (SNV) (Barnes et al. 1989), Savitzky-Golay (SG) smoothing (Savitzky et al. 1964), multiplicative scatter correction (MSC) (Hopke et al. 2003), and first derivate (Rinnan et al. 2009).

Due to undesired destruction of the test sample, previous NIR models which can be used in detection of gossypol in cottonseed meal, can be barely applied in breeding trails (Li et al. 2017). In this present study, spectroscopy was investigated the feasibility of analyzing gossypol in intact cottonseeds based on NIR spectrometer. The main aim of this study was to establish an optimal model which could provide a powerful technical support for cotton breeders and other people who work on cottonseeds.

\section{Materials And Methods}

\section{Samples and preparation}

A total of 268 samples of cottonseeds were collected from different growing areas, including Hangzhou (Zhejiang, China), Xiaoshan (Zhejiang, China), Sanmen (Zhejiang, China), Sanya (Hainan, China), Wuhu 
(Anhui, China), and Yancheng (Jiangsu, China), in 2012, 2013, and 2014. The cottonseed samples were delinted and dried at $30^{\circ} \mathrm{C}$ to constant weight. After spectral acquisition by NIR spectroscopy, the intact cottonseed samples were dehulled, and then ground to cottonseed kernel powder for HPLC analysis. The preparations were implemented in the same experimental condition in order to reduce the influence of other physical factors.

\section{Gossypol extraction}

$0.1 \mathrm{~g}$ of cottonseed kernel powder was suspended in $5 \mathrm{~mL}$ acetone and sonicated in an ultrasonic bath for 45 minutes. Then, the suspension was filtered through quantitative filter paper followed by a filtration with a $0.45 \mu \mathrm{m}$ syringe filter (Agela, Newark, USA). The sediment was washed three times by acetone. After this procedure, the extract was adjusted to $25 \mathrm{~mL}$ using acetone.

\section{HPLC analysis}

HPLC analysis was performed on an Agilent 1100 HPLC system (Agilent, Santa Clara, USA), equipped with an auto-sampler and UV detection. $\mathrm{A} \mathrm{C}_{18}$ column $(250 \mathrm{~mm} \times 4.6 \mathrm{~mm}, 5 \mu \mathrm{m}$, Dikma, Richmond Hill, USA) was employed as the stationary phase. The mobile phase consisted of methanol/ $0.2 \%$ $\mathrm{H}_{3} \mathrm{PO}_{4}(80 / 20, \mathrm{v} / \mathrm{v})$. The injection volume was $10 \mu \mathrm{L}$ and the flow rate was $1.0 \mathrm{~mL} \cdot \mathrm{min}^{-1}$. The $\mathrm{UV}$ detector was set at $238 \mathrm{~nm}$ and the column temperature was $25^{\circ} \mathrm{C}$. Each sample was measured three times. The limit of detection (LOD) was obtained at a signal-to-noise $(\mathrm{S} / \mathrm{N})$ ratio of three and the limit of quantification (LOQ) at an $\mathrm{S} / \mathrm{N}$ ratio. To detect the stability of gossypol at room temperature, three samples were randomly employed to determine the changes of peak area within 36 hours. HPLC-grade gossypol was purchased from Sigma (Sigma-Aldrich, St. Louis, USA). Methanol (HPLC grade) was procured from Tianjin Chemical Reagent Company (Tianjin, China). Double deionized water was prepared using Milli-Q-water purification system (Millipore, Molsheim, France).

\section{NIR spectra acquisition}

The NIR spectra of intact cottonseed samples were scanned with a Büchi Flex-N500 NIR spectrometer (Büchi, Flawil, Switzerland), equipped with a solid sample module as followings. The NIR spectra were collected across the range $4000-10000 \mathrm{~cm}^{-1}$, and were recorded with a spectral resolution of $4 \mathrm{~cm}^{-1}$. Samples were measured three times on a rotating cylinder device at $25 \pm 0.5^{\circ} \mathrm{C}$ and $60 \%$ relative air humidity. All the spectra were transformed into absorbance $(\log (1 / R))$. 


\section{Spectral pretreatment}

Before calibration, the spectral data were pretreated for an optimal performance. Eight pretreatment strategies which included one or some combination of Savitzky-Golay smoothing, SNV, MSC, and first derivate (Norris gap) were compared with the raw spectra.

\section{Sampling design}

Samples were assigned to calibration and prediction sets using Kennard-Stone (KS) selection (Kennard et al. 1969). The calibration models were established with the calibration set, and the prediction set was used to validate the predictive capabilities and analytical features of the calibration models.

\section{PLS regression}

PLS regression has been widely used as a calibration method to investigate the relationship between the spectral and the corresponding reference data. Before calibration of the PLS models, the data sets (spectral and reference data) were analyzed using 4-fold cross-validation to develop a full-spectra calibration model. The aim of the cross-validation was to find the optimum number of latent variables (LV) for PLS. The root-mean-square error of cross-validation (RMSECV) served as a measure to adjust the parameters, and the number of LV which provide the lowest RMSECV was selected as the best.

\section{Model evaluation}

The estimate of the calibration models was based on following quality parameters:

where $N$ is the total number of samples, $Y_{\text {nirs }}$ is the predicted value by calibrationmodels, $Y_{\text {ref }}$ is the reference value by HPLC, and SD is the standard deviation.

The coefficient for determination of prediction $\left(R_{\mathrm{p}}{ }^{2}\right)$, the root mean square error of prediction (RMSEP), the coefficient for determination of calibration $\left(R_{\mathrm{c}}{ }^{2}\right)$, the root mean square error of cross-validation (RMSECV), and the residual predictive deviation (RPD) were used as criterion to evaluate model performance. An acceptable model should have high $R_{\mathrm{c}}{ }^{2}$ and $R_{\mathrm{p}}{ }^{2}$ values and low RMSECV and RMSEP values. Meanwhile, the model is considered as robust if the RPD is higher than 2.5 .

\section{Software}

NIR spectroscopic data (268 samples $\times 1501$ variables) were exported in text format, organized in Excel spreadsheets, and then transferred into MATLAB R2011a (Math Works, Natick, USA) for chemometric 
analysis. All the algorithms in spectral pretreatments, sampling design, and regressions were implemented with MATLAB R2012a.

\section{Results}

\section{HPLC analysis}

The regression equation, correlation coefficient $\left(r^{2}\right)$, limits of detection (LOD), limits of quantification (LOQ), and average recovery of gossypol were illustrated in Table 1. The retention times of gossypol standard and gossypol extraction were 9.91 and 9.60 minutes, respectively (Fig. 1). Table 2 shows the stability for peak area of gossypol determined by HPLC for 24 hours. All the results indicated that the improved HPLC method could be used to detect gossypol content, and the cottonseed extract should be analyzed within 24 hours.

\section{Table 1}

HPLC-VU results.

\begin{tabular}{ccccc} 
sion equation & $r^{2}$ & LOD $\left(\mu \mathrm{g} \cdot \mathrm{mL}^{-1}\right)$ & LOQ $\left(\mu \mathrm{g} \cdot \mathrm{mL}^{-1}\right)$ & Average recovery (\%) \\
\hline$. .42 \mathrm{x}-85.055$ & 0.999 & 37.5 & 125.0 & $95.16-101.72$ \\
\hline
\end{tabular}

$r^{2}$, correlation coefficient; LOD, limits of detection; LOQ, limits of quantification; $\mathrm{y}$, peak area; $\mathrm{x}$, concentration $\left(\mu \mathrm{g} \cdot \mathrm{mL}^{-1}\right)$.

\section{Table 2}

The stability of gossypol determined for HPLC during $24 \mathrm{~h}$.

\begin{tabular}{|c|c|c|c|c|c|c|c|c|c|c|}
\hline sle & & & & & Time (h) & & & & & $R S D(\%)$ \\
\hline ier & 0 & 3 & 6 & 9 & 12 & 15 & 18 & 21 & 24 & \\
\hline & 2378.8 & 2398.0 & 2397.9 & 2435.2 & 2414.2 & 2421.1 & 2428.1 & 2452.7 & 2465.3 & 1.14 \\
\hline & 2871.7 & 2848.1 & 2860.2 & 2885.0 & 2876.6 & 2886.2 & 2932.9 & 2939.8 & 3010.5 & 1.76 \\
\hline & 2854.8 & 2839.8 & 2862.2 & 2892.6 & 2888.7 & 2918.6 & 2965.1 & 2972.7 & 3007.1 & 2.01 \\
\hline
\end{tabular}

$R S D$, relative standard deviation.

\section{NIR spectra analysis}

Across the spectral range of $4000-10000 \mathrm{~cm}^{-1}$, absorbance values are mainly associated with the combination and overtone bands of the $\mathrm{C}-\mathrm{H}, \mathrm{N}-\mathrm{H}, \mathrm{O}-\mathrm{H}$, and S-H bonds (Macho et al. 2002), which were quite sensitive to the compositional variations in complex samples. Fig. $2 \mathrm{~A}$ shows the raw intact 
cottonseed spectra in the NIR spectral region. The spectra showed six broad absorption peaks around the 4 200, 4 700, 5 150, 5 580, 6900 , and $8400 \mathrm{~cm}^{-1}$. The small peak observed at $4200 \mathrm{~cm}^{-1}$ fell within the regions associated with the combination bands of C-H. At 5150 and $6900 \mathrm{~cm}^{-1}$, these could be attributed to the combination and the first overtone bands of $\mathrm{O}-\mathrm{H}$, respectively, which were identified as water absorption. The gentle peaks at 5580 and $8400 \mathrm{~cm}^{-1}$ overlapped with the second and first $\mathrm{C}-\mathrm{H}$ overtone regions, respectively. It was worth mentioning that the peak at $4700 \mathrm{~cm}^{-1}$ was attributed to the first $\mathrm{C}-\mathrm{H}$ combination bands of alkenes and aromatic hydrocarbons, which could be identified as the absorption of polyphenolic terpenes, including gossypol and its derivatives.

The raw spectra were homogeneous, so the presence of noise could not be directly identified. Consistent baseline offsets and bias were present in the spectra, which are common features in the NIR spectra. Hence, eight pretreatment strategies were performed to optimize the raw spectra before establishment of the calibration models. The pretreatment spectra of several types of representative strategies were shown in Fig. 2B, Fig. 2C, and Fig. 2D. To different degrees, all these pretreatments could reduce the physical change among samples due to scattering and remove both additive and multiplicative effects in the spectra. It was noted that ten variables were lost after SG smoothing. Hence, the 1491 variables were used for calibration among the models using SG smoothing during the spectral pretreatments.

\section{Kennard-Stone sampling design}

The Kennard-Stone algorithm is an effective method for extracting a sample subset in multidimensional space, which includes all the most diverse samples and enables the selection of a subset of representative samples. Therefore, it has been confirmed that a calibration set extracted using KS selection has a better predictive capability than a set randomly built or constructed by other data selection methods such as Kohonen self-organized mapping (Kohonen et al. 1982) and D-optimal designs (de Aguiar et al. 1995). In this study, the total 268 intact cottonseed samples were divided into calibration and prediction sets based on KS algorithm, with the former set consisting of 218 samples and the later one 50 samples. The statistical values of gossypol contents in all cottonseed samples for calibration and prediction set were demonstrated in Table 3, which indicated that the range of variation for gossypol content was broad enough to develop NIR calibration models.

\section{Table 3}

Statistical values of gossypol content for calibration and prediction set samples.

\begin{tabular}{lccccc}
\hline a sets & $\mathrm{N}$ & Minimum $\left(\mathrm{g} \cdot \mathrm{kg}^{-1}\right)$ & Maximum $\left(\mathrm{g} \cdot \mathrm{kg}^{-1}\right)$ & Mean $\left(\mathrm{g} \cdot \mathrm{kg}^{-1}\right)$ & $\mathrm{SD}$ \\
\hline ation set & 218 & 0.32 & 1.04 & 0.63 & 0.16 \\
:tion set & 50 & 0.35 & 0.95 & 0.65 & 0.15 \\
\hline tmples & 268 & 0.32 & 1.04 & 0.64 & 0.16 \\
\hline
\end{tabular}

$\mathrm{N}$, number of samples; SD, standard deviation. 


\section{PLS regression}

The calibration models of gossypol content in intact cottonseeds based on PLS regression were established in the NIR spectral range of $4000-10000 \mathrm{~cm}^{-1}$, and the results were summarized in Table 4 . The number of LV were selected with the aid of cross-validation using the first minimum RMSECV for all models. The RMSECV and RMSEP values for all the calibration models were between 0.05-0.07 and 0.040.06 for calibration and prediction sets, respectively. The values of $R_{\mathrm{p}}{ }^{2}$ and $R_{\mathrm{c}}{ }^{2}$ ranged from 0.82 to 0.93 and from 0.87 to 0.97 , respectively. The RPD values ranged from 2.3 to 3.4 .

\section{Table 4}

Performance comparison results for calibration models using different spectral pretreatment strategies.

\begin{tabular}{ccccccc}
\hline ectral pretreatments & LVs & \multicolumn{2}{c}{ Calibration set } & \multicolumn{3}{c}{ Prediction set } \\
\cline { 2 - 7 } & & RMSECV & $R_{\mathrm{C}}{ }^{2}$ & RMSEP & $R_{\mathrm{p}}{ }^{2}$ & $\mathrm{RPD}$ \\
\hline Raw & 9 & 0.06 & 0.94 & 0.06 & 0.82 & 2.3 \\
\hline SG & 9 & 0.07 & 0.87 & 0.06 & 0.86 & 2.5 \\
\hline SNV & 10 & 0.06 & 0.92 & 0.05 & 0.87 & 2.8 \\
\hline MSC & 8 & 0.06 & 0.92 & 0.05 & 0.88 & 2.8 \\
\hline 1st D & 9 & 0.05 & 0.95 & 0.05 & 0.87 & 2.7 \\
\hline SNV+1st D & 10 & 0.06 & 0.96 & 0.05 & 0.89 & 3.0 \\
\hline MSC+1st D & 9 & 0.06 & 0.96 & 0.05 & 0.88 & 2.9 \\
\hline SG+SNV+1st D & 9 & 0.05 & 0.97 & 0.04 & 0.92 & 3.4 \\
\hline SG+MSC+1st D & 10 & 0.05 & 0.96 & 0.05 & 0.89 & 3.0 \\
\hline
\end{tabular}

LVs, latent variables; RMSECV, root mean square error of cross-validation; $R_{\mathrm{C}}{ }^{2}$, coefficient of determination of calibration; RMSEP, root mean square error of prediction; $R_{\mathrm{p}}{ }^{2}$, coefficient of determination of prediction; RPD, residual predictive deviation; Raw, raw spectra; SG, Savitzky-Golaysmoothing; SNV, standard normal variate; MSC, multiplicative scatter correction; 1st D, first derivate.

\section{Discussion}

Since NIR spectra of intact cottonseeds were complex and overlapped, suitable spectral pretreatments should be used to optimize the NIR spectra and extract the effective information. In this work, the raw spectra were transformed using by eight pretreatment strategies, including the singe pretreatment 
strategies (SG smoothing, SNV, MSC, and first derivate), two pretreatments strategies (SNV + first derivate and MSC + first derivate), and three pretreatments strategies (SG smoothing + SNV + first derivate and SG smoothing + MSC + first derivate). In analyzing the results obtained from singe pretreatment strategies, the PLS model using eight latent variables based on application of MSC produced better results with low values of RMSECV and RMSEP ( 0.06 and 0.05 , respectively), and the RPD value was increased by $20.36 \%$ compared with that of the direct regression model based on raw spectra (Fig. 4). Fig. 3B shows the correlation of model using MSC, presented by plotting predicted and reference values for gossypol content in intact cottonseeds. The samples near the diagonal line indicated that their predicted values were more closed to reference ones and vice versa. In the aspect of two pretreatments strategies, the calibration model based on SNV + first derivate, presented a better predictive ability than that on MSC + first derivate, with the $R_{\mathrm{c}}{ }^{2}$ and $R_{\mathrm{p}}{ }^{2}$ values of 0.962 and 0.887 , respectively. The RPD value of that model was 3.0 , increased by $28.14 \%$ compared to the model using raw spectra. From all the results of calibration models established, the best model was pretreated using the strategy of SG + SNV + first derivate, and it had the highest $R_{\mathrm{c}}{ }^{2}(0.97)$ and $R_{\mathrm{p}}{ }^{2}(0.93)$, and the RPD (3.4) increased by $46.28 \%$ compared with that of raw spectral model. Furthermore, RMSECV (0.05) and RMSEP (0.04) were the lowest among all the models. The correlation plots between predicted and reference values were focused on the diagonal line (Fig. 3D). It was indicated that the model using SG + SNV + first derivate and PLS was accurate and robust enough to substitute the conventional gossypol analysis methods (HPLC) to measure gossypol in intact cottonseeds.

The NIR spectra of these intact seeds generally contained a mass of undesirable features, including noise, overlapping peaks, baseline effects and some systematic behaviors, caused by the seed size, shell, and some other physical factors. Hence, a suitable pretreatment strategy was required for widespread application of NIR technology in crop seed analysis. In this work, it was indicated that an advisable pretreatment strategy before regression was important to refine the effective information from spectral data and eliminate spectral deviation to calibrate an accurate and robust NIR model.

The calibration models reported here confirmed the feasibility of the using of NIR technology for rapid and non-destructive determination of gossypol, an important parameter to cottonseed products, in intact cottonseeds for the first time. The high RPD values (3.4) suggested that this technology could be an effective method for the measurement of gossypol in intact cottonseeds. The optimal model could substitute conventional analysis methods for gossypol, including UV spectrophotometry and HPLC. Because of the potential of high sample throughput and low costs, as well as a significant reduction in toxic chemicals, the application of NIR method could be encouraged and popularized to other similar agricultural products.

\section{Conclusions}

The calibration and validation statistics obtained in the current work showed the potential of NIRS to predict microelement gossypol content in intact cottonseeds. The optimized model was that pretreated by 
Savitzky-Golay smoothing + standard normal variate + first derivate, with RMSECV, RMSEP, $R_{\mathrm{p}}{ }^{2}$, and RPD of $0.05,0.04,0.92$, and 3.4 , respectively, which provided a method to determine gossypol content in intact cottonseeds feasibly.

\section{Declarations}

\section{Ethics approval and consent to participate}

Not applicable.

\section{Consent for publication}

All co-authors have consent for submission of manuscript.

\section{Availability of data and materials}

All relevant data are within this article.

\section{Competing interests}

The authors declare that they have no competing interests.

\section{Funding}

The research work was funded by The National Key Technology R\&D program of China (2016YFD0101404), China Agriculture Research System (CARS-18-25), and Jiangsu Collaborative Innovation Center for Modern Crop Production.

\section{Author's contributions}

Li C (Cheng) and Zhu SJ designed the experiments and wrote the manuscript. Li C (Cheng), Zhao TL and Su BS analyzed the data, Li C(Cheng), SU BS, Li C (Cong) participated in experiment. Chen JH assisted in editing the article. Zhu SJ and Chen JH conducted and supervised the experiments.

\section{Acknowledgments}

We are grateful to Mrs Yu Liu for her technical assistance.

\section{References}

Bala $\mathrm{M}$ and Singh M. Non-destructive estimation of total phenol and crude fiber content in intact seeds of rapeseed-mustard using FTNIR. Industrial Crop and Products. 2013; 42:357-362.

Barnes R, Dhanoa M and Lister S. Standard normal variate transformation and de-trending of nearinfrared diffuse reflectance spectra. Applied Spectroscopy. 1989; 43:772-777. 
Bellato S, Frate DV, Redaelli R, et al. Use of near infrared reflectance and transmittance coupled to robust calibration for the evaluation of nutritional value in naked oats. Journal of Agricultural Food and Chemistry. 2011; 59:4349-4360.

Blanco A, Aoki A, Montamat E, et al. Effect of gossypol upon motility and ultrastructure of Trypanosoma cruzi. The Journal of Protozoology. 1983; 30: 649-651.

de Aguiar PF, Bourguignon B, Khots MS, et al. D-optimal designs. Chemometrics and Intelligent Laboratory Systems. 1995; 30:199-210.

Fassio A and Cozzolino D. Non-destructive prediction of chemical composition in sunflower seeds by near infrared spectroscopy. Industrial Crop and Products. 2004; 20:321-329.

Haaland MD and Thomas VE. Partial least-squares methods for spectral analyses. 1. Relation to other quantitative calibration methods and the extraction of qualitative information. Analytical Chemistry. 1988; 60:1193-1202.

Hacisalihoglu G, Larbi B and Settles A. Near-infrared reflectance spectroscopy predicts protein, starch, and seed weight in intact seeds of common bean (Phaseolus vulgaris L.). Journal of Agricultural Food and Chemistry. 2010; 58:702-706.

Huang ZR, Sha S, Rong ZQ, et al. Feasibility study of near infrared spectroscopy with variable selection for non-destructive determination of quality parameters in shell-intact cottonseed. Industrial Crop and Products. 2013; 43:654-660.

Kennard RW and Stone LA, Computer aided design of experiments. Technometrics. 1969; 11:137-148.

Kohonen T, Analysis of a simple self-organizing process. Biological Bybernetics. 1982; 44:135-140.

Kong GC, Daud KM and SJ Z. Effects of pigment glands and gossypol on growth, development and insecticide-resistance of cotton bollworm (Heliothis armigera (Hübner)). Crop Protection. 2010; 29:813819.

Kovalenko VI, Rippke RG and Hurburgh RC. Determination of amino acid composition of soybeans (Glycine max) by near-infrared spectroscopy. Journal of Agricultural Food and Chemistry. 2006; 54:34853491.

Hopke H. The evolution of chemometrics. Analytica Chimica Acta. 2003; 500:365-377.

Lee $\mathrm{H}$, Kim M, Song Y, et al. Non-destructive evaluation of bacteria-infected watermelon seeds using visible/near-infrared hyperspectral imaging. Journal of Science and Food Agriculture. 2017; 97:10841092. 
Li C, Zhao TL, Li C, et al. Determination of gossypol content in cottonseeds by near infrared spectroscopy based on Monte Carlo uninformative variable elimination and nonlinear calibration methods. Food Chemistry. 2017; 221:990-996.

Lin C, Chen X, Jian L, et al. Determination of grain protein content by near-infrared spectrometry and multivariate calibration in barley. Food Chemistry. 2013; 162:10-15.

Lin TS, Schinazi RF, Zhu JL, et al. Anti-Hiv-1 activity and cellular pharmacology of various analogs of gossypol. Biochemical Pharmacology. 2013; 46:251-255.

Lordelo M.M, Davis AJ, Calhoun MC, et al. Relative toxicity of gossypol enantiomers in broilers. Poultry Science. 2005; 84:1376-1382.

Macho S and Larrechi MS, Near-infrared spectroscopy and multivariate calibration for the quantitative determination of certain properties in the petrochemical industry. Trends in Analytical Chemistry. 2002; 21:799-806.

Makinoa Y, Ichimura M, Oshita S, et al. Estimation of oxygen uptake rate of tomato (Lycopersicon esculentum Mill.) fruits by artificial neural networks modelled using near-infrared spectral absorbance and fruit mass. Food Chemistry. 2010; 121:533-539.

Mendoza AF, Cichy AK, Sprague C, et al. Prediction of canned black bean texture (Phaseolus vulgaris L.) from intact dry seeds using visible/near-infrared spectroscopy and hyperspectral imaging data. Journal of Science and Food Agriculture. 2018; 98:283-290.

Nie Z, Han J, Liu T and Liu X. Hot topic: Application of support vector machine method in prediction of alfalfa protein fractions by near infrared reflectance spectroscopy. Journal of Dairy Science. 2008; 91:2361-2369.

Rinnan $\AA$, van den Berg F and Engelsen SB. Review of the most common pre-processing techniques for near-infrared spectra. Trends in Analytical Chemistry. 2009; 28:1201-1222.

Rosales A, Galicia L, Oviedo E, et al. Near-infrared reflectance spectroscopy (NIRS) for protein, tryptophan, and lysine evaluation in quality protein maize (QPM) breeding programs. Journal of Agricultural Food and Chemistry. 2011; 59:10781-10786.

Savitzky A and Golay M. Smoothing and differentiation of data by simplified least squares procedures. Analytical Chemistry. 1964; 36:1627-1639.

Sawan MZ, Hafez AS, Basyony EA, et al. Cottonseed, protein, oil yields and oil properties as affected by nitrogen fertilization and foliar application of potassium and a plant growth retardant. World Journal of Agricultural Science. 2006; 1:56-65. 
Shao YN, Zhao CJ, Bao YD, et al. Quantification of nitrogen status in rice by least squares support vector machines and reflectance spectroscopy. Food and Bioprocess Technology. 2012; 5:100-107.

Sohn M, Himmelsbach SD, Barton EF, et al. Near-infrared analysis of whole kernel barley: comparison of three spectrometers. Applied Spectroscopy. 2008; 62:427-432.

Sunilkumar G, Campbell CL, Puckhaber L, et al. Engineering cottonseed for use in human nutrition by tissue-specific reduction of toxic gossypol. Proceedings of the National Academy of Science. 2006; 103:18054-18059.

Tierno R, López A, Riga P, et al. Phytochemicals determination and classification in purple and red fleshed potato tubers by analytical methods and near infrared spectroscopy. Journal of Science and Food Agriculture. 2016; 96:1888-1899.

Weinstock A, Janni J, Hagen L, et al. Prediction of oil and oleic acid concentrations in individual corn (Zea mays $\mathrm{L}$.) kernels using near-infrared reflectance hyperspectral imaging and multivariate analysis. Applied Spectroscopy. 2006; 60:9-16.

Xie YL and Kalivas HJ. Local prediction models by principal component regression. Analytica Chimica Acta. 1997; 348:29-38.

Yang $\mathrm{N}$ and Ren QX. Application of near-infrared reflectance spectroscopy to the evaluation of rutin and d-chiro-inositol contents in tartary buckwheat. Journal of Agricultural Food and Chemistry. 2008; 56:761764.

\section{Figures}



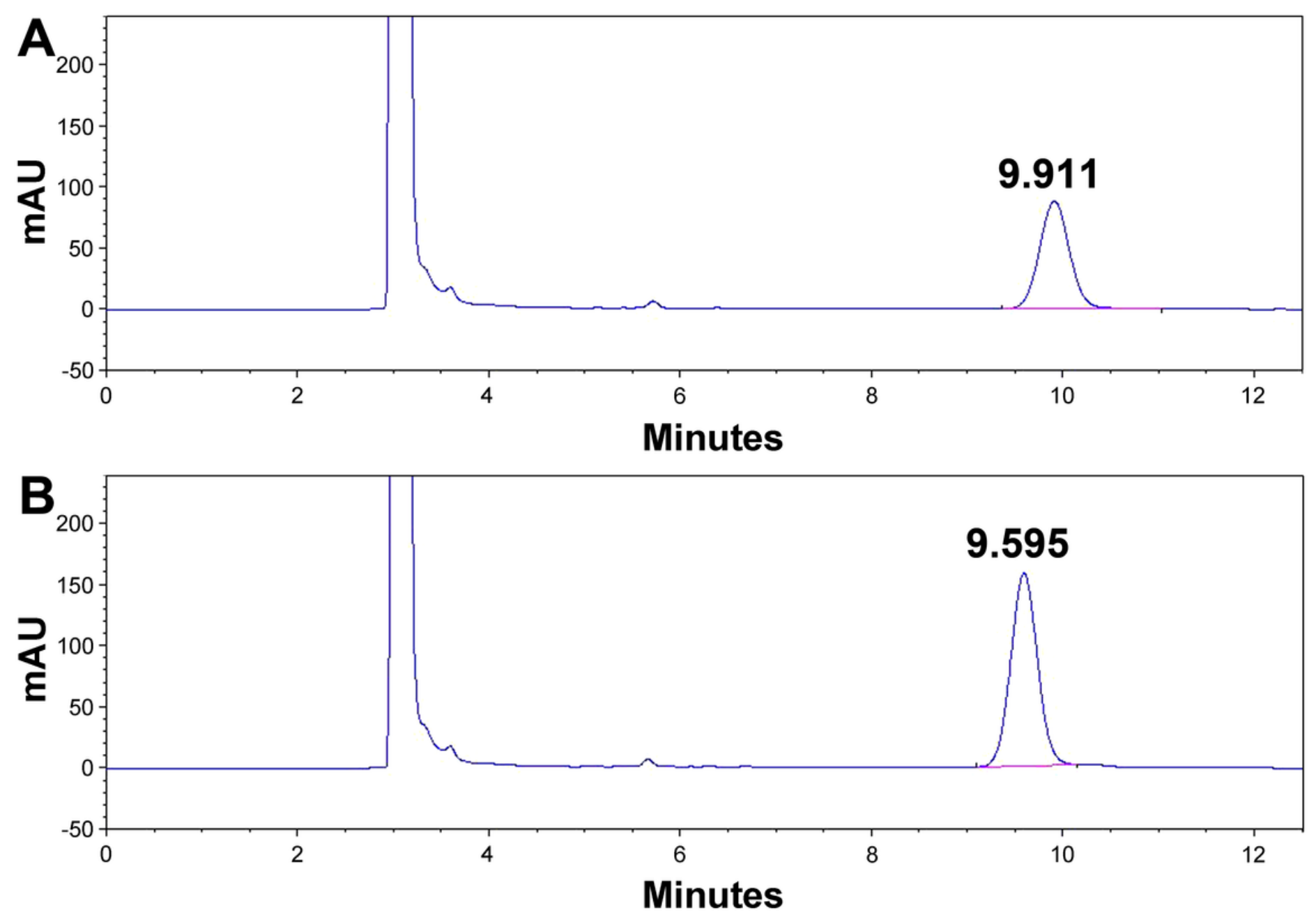

Figure 1

Chromatograms of (A) gossypol standard and (B) gossypol extract in intact cottonseeds. 

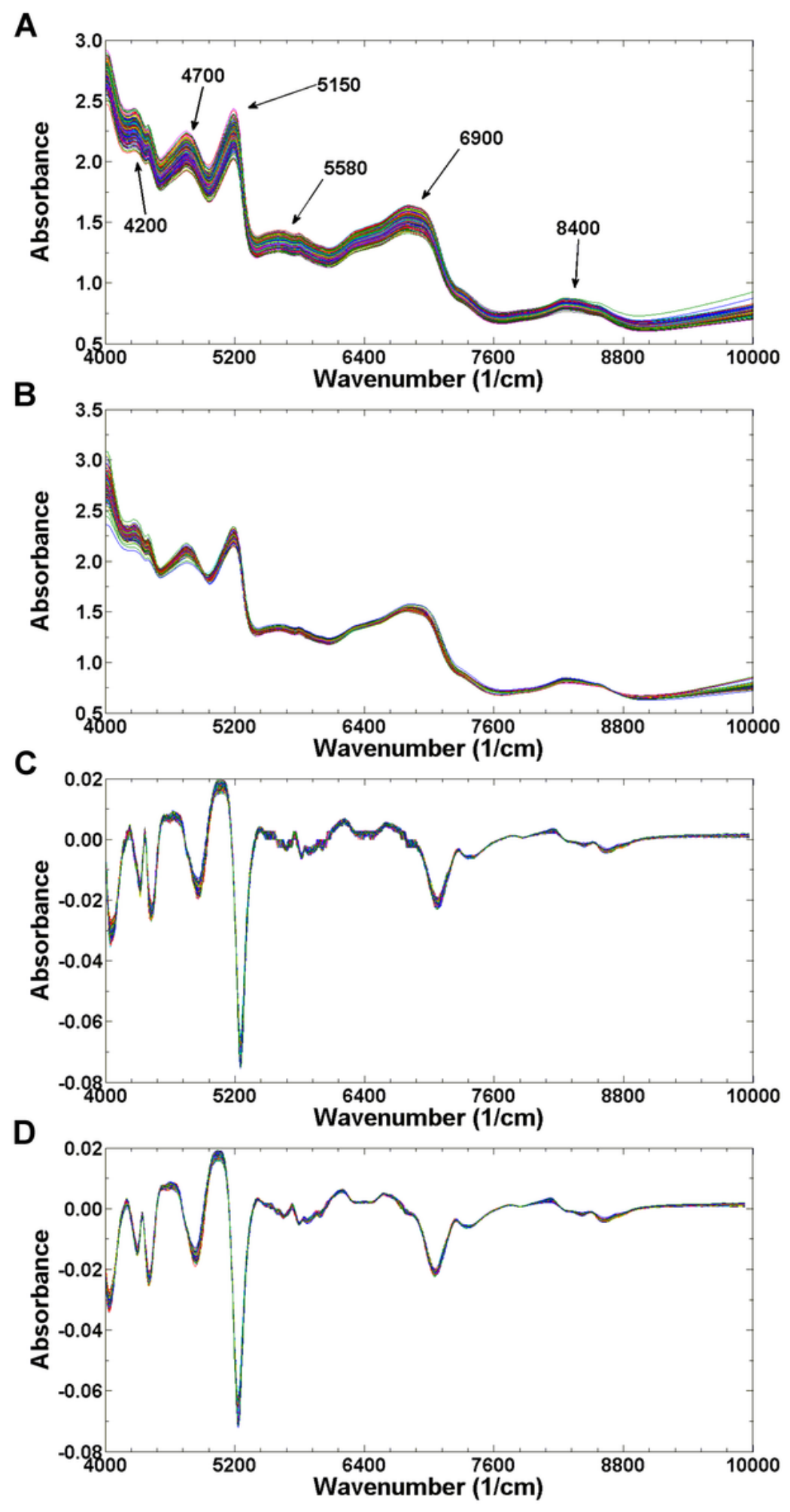

\section{Figure 2}

The NIR spectra of intact cottonseeds. (A) The raw spectra, (B) the spectra pretreated by MSC, (C) the spectra pretreated by SNV+ first derivate, and (D) the spectra of pretreated by SG smoothing + SNV+ first derivate. 

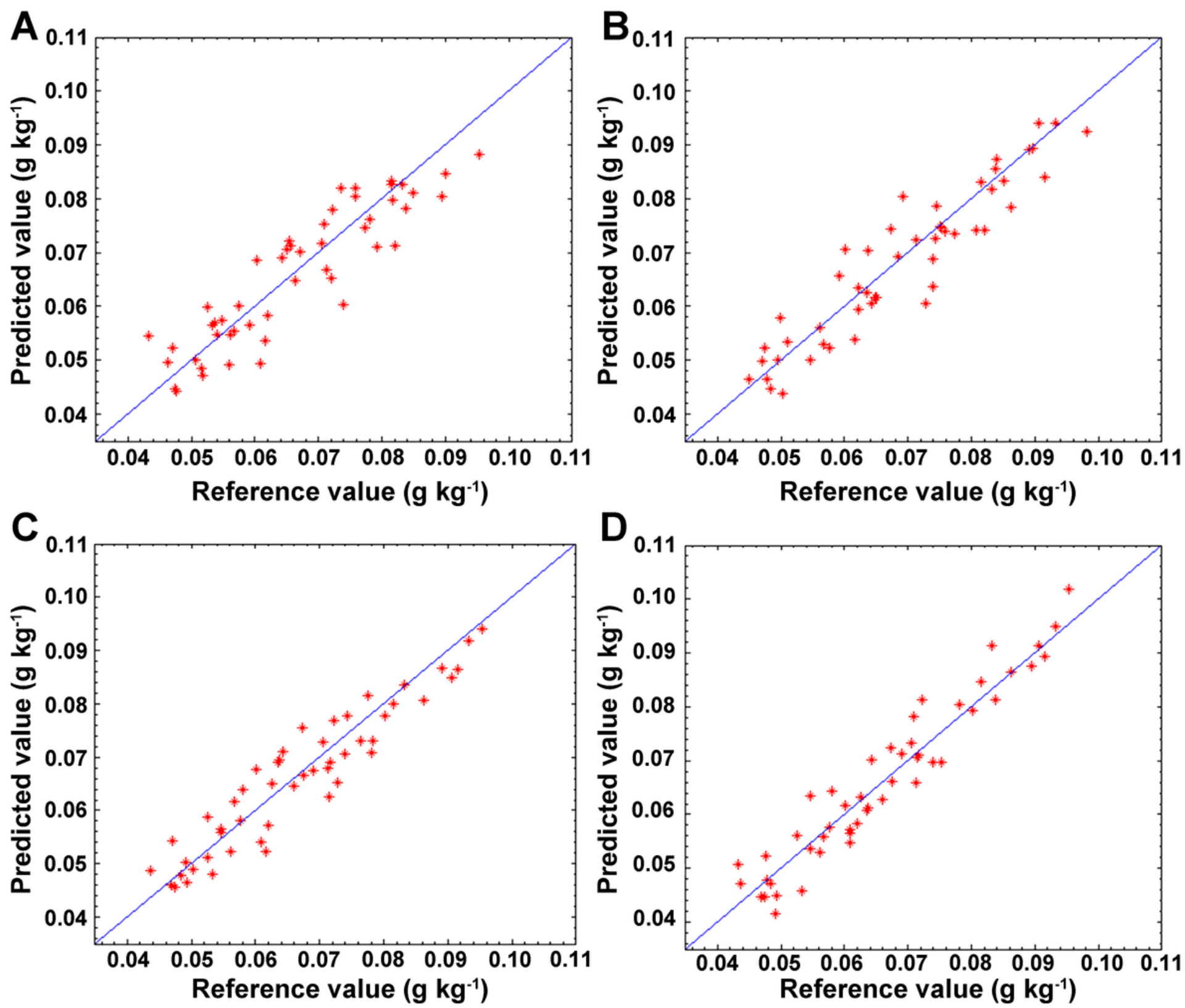

Figure 3

The correlation between predicted and reference values for models of intact cottonseeds. (A) the PLS model based on raw spectra, (B) the PLS model based on the pretreatment of MSC, (C) the PLS model based on the pretreatment of SNV+ first derivate, and (D) the PLS model based on the pretreatment of SG smoothing+ SNV+ first derivate. 


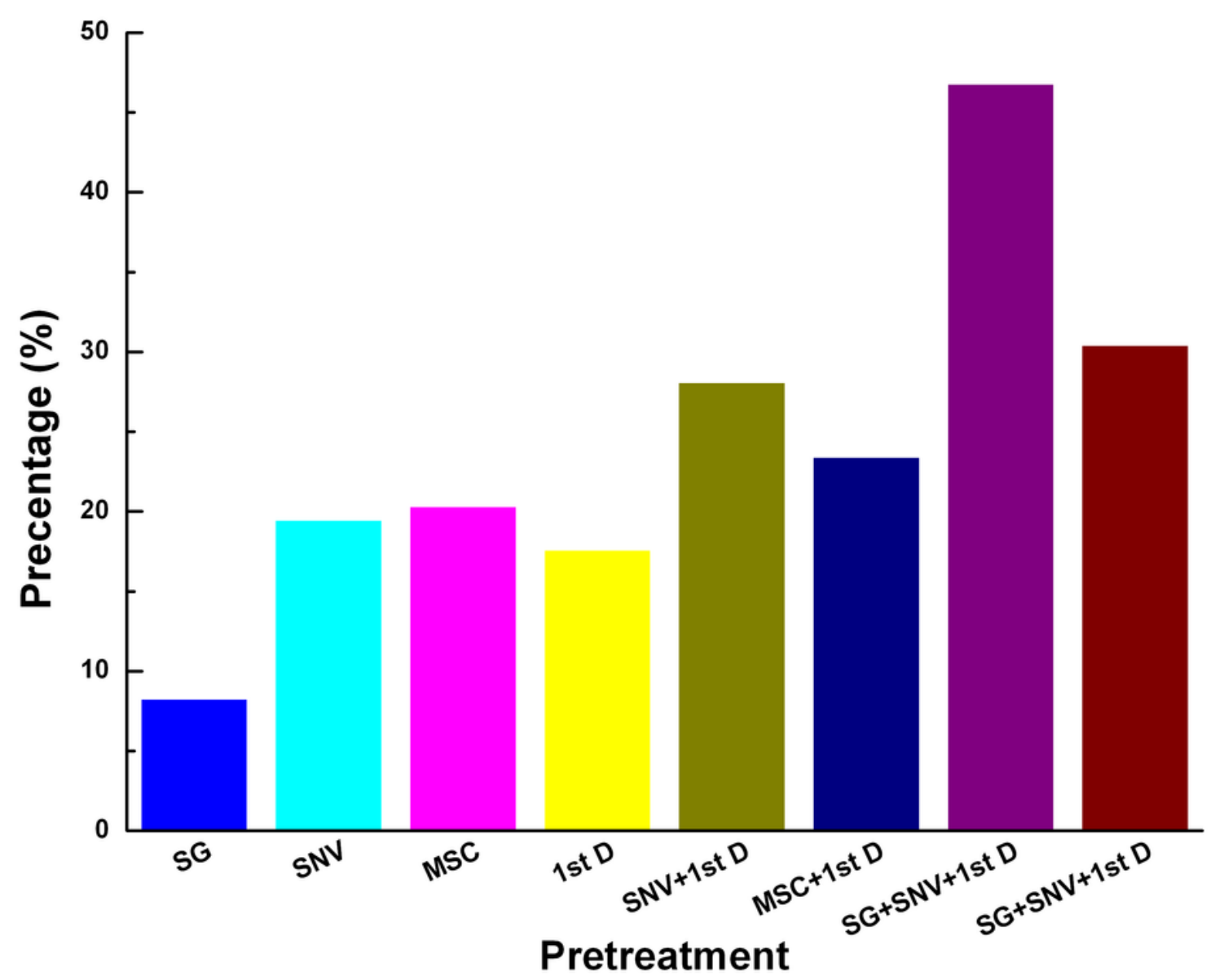

Figure 4

The residual predictive deviation (RPD) for PLS models based on different pretreatment strategies compared with the model using raw spectra. 